This is a postprint version of the following published document:

Baselga, J., Hernández-Fuentes, I., Piérola, I. F. \& Llorente, M. A. (1987). Elastic properties of highly crosslinked polyacrylamide gels. Macromolecules, 20 (12), pp. 3060-3065.

“ This document is the unedited Author's version of a Submitted Work that was subsequently accepted for publication in Macromolecules, copyright (C) 1987 American Chemical Society after peer review. To access the final edited and published work see http://pubs.acs.org/doi/abs/10.1021/ma00178a020, or see DOI: $10.1021 / \mathrm{ma} 00178 \mathrm{a} 020 "$ 


\title{
Elastic Properties of Highly Cross-Linked Polyacrylamide Gels
}

\author{
J. Baselga, ${ }^{\dagger}$ I. Hernández-Fuentes ${ }^{\dagger}$ I. F. Piêrola,${ }^{\dagger}$ and M. A. Llorente ${ }^{* \ddagger}$ \\ Departamento Quimica Fïsica (I), Fac. CC. Quimicas, Universidad Complutense, 28040 Madrid, Spain, and Departamento Quimica \\ Fisica, Fac. Ciencias, Universidad a Distancia (UNED), 28040 Madrid, Spain.
}

${ }^{\dagger}$ Universidad Complutense. ${ }^{\ddagger}$ Universidad a Distancia.

\begin{abstract}
Mechanical properties of polyacrylamide gels covering a wide range of polymer concentrations have been studied. Gels were synthesized by using $\mathrm{N}, \mathrm{N}^{\prime}$-methylenebis(acrylamide) as cross-linking agent whose weight percentage, with respect to the total weight of comonomers, ranged from $0.663 \%$ to $14.50 \%$. The resulting gels were analyzed by means of their stress-strain isotherms in elongation at $30^{\circ} \mathrm{C}$. Mooney-Rivlin type plots of the data show a large increase of the modulus or upturn,

particularly on gels with high percentage of cross-linking monomer and at high polymer concentrations, due to non-Gaussian effects arising from the very heterogeneous molecular network structure. The elastic modulus was found to increase exponentially with total comonomer concentration, keeping constant the percentage of bisacrylamide comonomer. On the other hand, the modulus passes through a maximum as the amount of cross-linking agent is increased. The ultimate

properties found and the comparison of the cross-linking densities obtained from the elastic results with the theoretical ones, determined from the initial comonomer compositions, confirm the very high heterogeneity of f?Olyacrylamide gels.
\end{abstract}

\section{Introduction}

Polyacrylamide gels (PAA) are widely used in a large number of applications, both academic and technological. ${ }^{1}$ Their properties are determined by the molecular structure which is in turn a consequence of the gelation process. This process is usually carried out by cross-linking co polymerization of acrylamide (AA) with a small amount of a crosslinking monomer, such as $\mathrm{N}, \mathrm{N}^{\prime}$-methylenebis (acrylamide) (BA), in aqueous solution. -The resulting gel structure is strongly dependent on the total concentration of both comonomers (CT, g/100 mL), the crosslinking monomer percentage $(\mathrm{C}, \%)$ as well as some other factors which affect the gelation kinetics, such as solvent viscosity, temperature and initiator concentration. Several studies have shown that PAA gels are very heterogeneous, having a structure in which regions of very different degree of cross-linking coexist. ${ }^{2}$

Mechanical characteristics of PAA gels are very im portant properties for a number of practical applications. Several techniques such as dynamic light scattering,2 viscoelastic measurements, ${ }_{-5}{ }_{-5}$ stress-relaxation ${ }^{6}$ and swelling equilibrium ${ }^{7}$ have been used in order to determine the elastic modulus. Direct equilibrium stress-strain measurements are scarce due to experimental difficulties, arising from the very soft systems under consideration, and only a few experiments on compression have been re ported.8. 9 On the other hand most of the measurements have been carried out on gels of low values of the param eter $\boldsymbol{C}$, from which transparent gels are obtained. Therefore experiments covering a broader range of co monomer concentrations are needed.

In this paper we have studied the elastic behavior of a

large number of PAA gels covering a wide range of values of $\mathrm{C}_{\mathrm{T}}$ and $\boldsymbol{C}$. The gels were characterized by means of their stress-strain isotherms in elongation for which an exper imental method of avoiding problems due to the softness of the material has been developed. The two principal properties investigated were the elastic modulus and the ultimate properties, both being related to the molecular structure of the gels.

\section{Experimental Section}

Reagents. Acrylamide (AA) and $N, N^{\prime}$-methylenebis(acrylamide) (BA) were purchased from Eastman Kodak. BA was used after recrystallization from acetone. The redox pair potassium persulphate (PSK)/triethanolamine (TEA), both products from Carlo Erba, was used as the room temperature initiator system. Water was purified from salts and organic matter by a Milli-Q equipment Millipore, and filtered with a $0.45-\mu \mathrm{m}$ filter.

Sample Preparation. The usual preparation of samples for stress-strain measurements (cutting pieces of an adequate geometry starting from a bigger film) is not successful because of the brittleness of polyacrylamide (PAA) gels; it is very difficult to obtain samples with sharp borders and attach them to the transducer clamps.

In order to overcome these difficulties the samples were synthesized with their final geometry in a mold as shown in Figure 1. Two pieces of glass confine a hollowed out Teflon rectangle in which two small holes have been bored for the injection of the reagents in aqueous solution.

At the top and bottom of the mold two pieces of a woven material have been placed, which become impregnated with the comonomer solution when this is injected. When the gel is formed, its extremities have a great consistency and can be fastened easily. In all measurements that have been tested the textile has no influence on rupture because it always occurred outside the textile-gel boundary.

Gels with different compositions have been studied varying the total comonomer concentration, expressed as $C_{\mathrm{T}}(\mathrm{g} / 100 \mathrm{~mL})$, and the weight fraction of the cross-linker in the mixture, $C(\%)$. As shown in Figure 2, where the selected experiments are superimposed over the phase diagram reported by Bansil and Gupta, ${ }^{10}$ there are two sets of experiments where $C_{\mathrm{T}}$ and $C$ have been kept constant, $C_{\mathrm{T}} \simeq 6.7 \mathrm{~g} / 100 \mathrm{~mL}$ and $C \simeq 10 \%$.

In all cases initiator concentrations have been [TEA] $=8.51$ $\times 10^{3} \mathrm{M}$ and $[\mathrm{PSK}]=6.57 \times 10^{-3} \mathrm{M}$.

The polymerizations were carried out at room temperature for 2 days to ensure total conversion. Afterward the samples were immersed in water for a period of time between 2 and 5 days at $30^{\circ} \mathrm{C}$, in order to extract initiator residues and soluble parts of the networks and to attain swelling equilibrium prior to the elastic measurements. The network volume fractions have been determined on pieces of measured samples weighing them before and after they were vacuum-dried at $50^{\circ} \mathrm{C}$ until constant weight. The characteristics of the gels thus prepared are given in Table $\mathrm{I} ; v_{2 c}$ is the volume fraction of monomers in the reaction mixture and $v_{2 s}{ }^{0}$ is the volume fraction of polymer in the resulting gel, swollen at equilibrium, in the unstretched state.

The initial dimensions of the samples ( $L_{0}$ and cross-sectional area) were measured in a relaxed state of the samples, horizontally, to avoid the deformation self-induced by the weight of the gels. Cross-sectional areas of the swollen samples were approximately $70 \mathrm{~mm}^{2}$.

Stress-Strain Measurements. Elastic modulus of PAA gels has been determined from stress-strain measurements on gel samples immersed in pure water at swelling equilibrium at $30^{\circ} \mathrm{C}$. 
Table I

Characteristics and Elastic Results on PAA Gels

\begin{tabular}{|c|c|c|c|c|c|c|c|c|c|c|c|}
\hline gel & $C_{\mathrm{T}}, \mathrm{g} / 100 \mathrm{~mL}$ & $C, \% \mathrm{w} / \mathrm{w}$ & $v_{2 \mathrm{c}}$ & $v_{28}{ }^{\circ}$ & {$\left[f_{\mathrm{ph}}\right], \mathrm{N} \cdot \mathrm{mm}^{-2}$} & $\kappa$ & $\zeta$ & $10^{3} M_{\mathrm{c}}$ & $M_{\mathrm{c}}^{\exp } / M_{\mathrm{c}}^{\text {th }}$ & $\alpha_{\mathrm{r}}$ & $10^{-3} E_{\mathrm{r}}, \mathrm{J} \cdot \mathrm{m}^{-3}$ \\
\hline \multirow[t]{2}{*}{ T-1 } & 18.0 & 10.1 & 0.145 & 0.158 & 0.311 & 6.0 & 0.40 & 1.46 & 2.13 & 1.10 & 4.12 \\
\hline & & & & 0.156 & 0.336 & 5.7 & 0.40 & 1.40 & 2.03 & 1.10 & 4.24 \\
\hline \multirow[t]{2}{*}{$\mathrm{T}-2$} & 15.0 & 10.0 & 0.119 & 0.128 & 0.119 & 9.6 & 0.20 & 2.75 & 3.97 & 1.27 & 15.0 \\
\hline & & & & 0.126 & 0.150 & 8.6 & 0.15 & 3.60 & 5.19 & 1.28 & 13.0 \\
\hline \multirow[t]{2}{*}{ T-3 } & 11.0 & 9.5 & 0.0872 & 0.0903 & 0.0685 & 13.0 & 0.15 & 5.07 & 6.91 & 1.30 & 8.19 \\
\hline & & & & 0.0862 & 0.0723 & 12.0 & 0.15 & 4.67 & 6.36 & 1.27 & 6.69 \\
\hline \multirow{2}{*}{$\mathrm{T}-4$} & 7.67 & 10.2 & 0.0600 & 0.0587 & 0.0217 & 22.0 & 0.08 & 12.6 & 18.5 & 1.35 & 3.41 \\
\hline & & & & 0.0627 & 0.0265 & 20.0 & 0.07 & 10.0 & 14.8 & 1.48 & 7.14 \\
\hline \multirow[t]{2}{*}{ T-5 } & 6.72 & 10.1 & 0.0525 & 0.0487 & 0.0193 & 24.0 & 0.05 & 12.8 & 18.6 & 1.54 & 6.61 \\
\hline & & & & 0.0484 & 0.0170 & 25.0 & 0.06 & 14.8 & 21.6 & 1.64 & 8.79 \\
\hline \multirow[t]{2}{*}{ T-6 } & 4.99 & 10.0 & 0.0388 & 0.0388 & 0.0074 & 38.0 & 0.03 & 28.9 & 41.6 & 1.73 & 4.30 \\
\hline & & & & 0.0381 & 0.0072 & 39.0 & 0.026 & 29.3 & 42.3 & 1.53 & 2.14 \\
\hline \multirow{2}{*}{ C-1 } & 6.71 & 0.663 & 0.0515 & 0.0124 & 0.0026 & 65.0 & 0.04 & 88.9 & 7.7 & 2.43 & 4.82 \\
\hline & & & & 0.0138 & 0.0022 & 71.0 & 0.03 & 113.0 & 9.9 & 2.60 & 4.29 \\
\hline C-2 & 6.60 & 1.134 & 0.0515 & 0.0293 & 0.0093 & 35.0 & 0.18 & 24.3 & 3.6 & 2.21 & 12.9 \\
\hline \multirow{2}{*}{$\mathrm{C}-3$} & 6.70 & 2.599 & 0.0523 & 0.0303 & 0.0130 & 29.0 & 0.025 & 19.4 & 6.7 & 1.78 & 7.67 \\
\hline & & & & 0.0314 & 0.0138 & 28.0 & 0.035 & 19.5 & 6.7 & 1.72 & 7.34 \\
\hline C-4 & 6.69 & $3: 950$ & 0.0522 & 0.0403 & 0.0192 & 24.0 & 0.05 & 12.7 & 6.7 & 1.72 & 10.3 \\
\hline \multirow[t]{2}{*}{ C-5 } & 6.71 & 5.784 & 0.0524 & 0.0463 & 0.0165 & 26.0 & 0.04 & 15.4 & 12.2 & 1.55 & 5.54 \\
\hline & & & & 0.0443 & 0.0192 & 24.0 & 0.05 & 13.0 & 10.3 & 1.60 & 7.58 \\
\hline \multirow[t]{2}{*}{ C-6 } & 6.67 & 6.951 & 0.0521 & 0.0501 & 0.0195 & 23.0 & 0.06 & 12.7 & 12.3 & 1.43 & 4.11 \\
\hline & & & & 0.0571 & 0.0190 & 24.0 & 0.06 & 13.0 & 12.6 & 1.62 & 8.34 \\
\hline C-7 & 6.68 & 8.756 & 0.0522 & 0.0463 & 0.0154 & 27.0 & 0.06 & 16.1 & 20.1 & 1.38 & 2.72 \\
\hline \multirow[t]{2}{*}{ C-9 } & 6.70 & 11.91 & 0.0523 & 0.0475 & 0.0135 & 29.0 & 0.06 & 18.4 & 32.3 & 1.44 & 3.27 \\
\hline & & & & 0.0498 & 0.0135 & 29.0 & 0.05 & 18.4 & 32.3 & 1.58 & 5.78 \\
\hline \multirow[t]{2}{*}{ C-10 } & 6.80 & 13.98 & 0.0531 & 0.0460 & 0.0100 & 33.0 & 0.05 & 24.7 & 52.2 & 1.40 & 1.91 \\
\hline & & & & & 0.0108 & 33.0 & 0.05 & 23.4 & 49.4 & 1.44 & 2.55 \\
\hline C-11 & 6.67 & 14.50 & 0.0521 & 0.0487 & 0.0103 & 33.0 & 0.05 & 24.4 & 53.6 & 1.31 & 1.21 \\
\hline
\end{tabular}

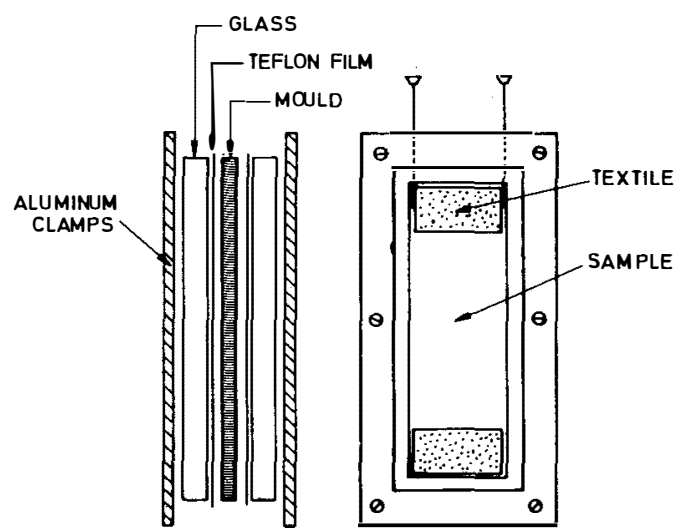

Figure 1. Scheme of the mold used for samples preparation.

The general features of the apparatus have been described elsewhere. ${ }^{11}$ The force transducer (HBM VI) has been selected in order to measure the very low initial forces, approximately 0.02 $\mathrm{N}$, being recorded with a graphic recorder (J.J. Instruments CR600) working at its maximum sensibility $(1 / 20 \mathrm{mV} / \mathrm{cm})$. With these recording conditions a signal drift less than $1-2 \%$ over a 24-h period has been found.

The samples were immersed in water inside a glass vessel provided with a constant-temperature water jacket. The temperature inside the vessel was checked with a digital thermometer with its probe located near the sample. The strain was followed measuring the distance between two ink points with a cathetometer (Ealing 11-5253).

The relaxation delay between successive experimental points depends not only on the relaxation of the sample but on the new swelling equilibrium that must be attained, which is a function of the cross-linking density and was found to be between 2 and $6 \mathrm{~h}$.

\section{Results and Discussion}

The elastic results have been interpreted by means of the inodulus or "reduced force", which is defined by ${ }^{12}$

$$
\left[f^{*}\right]=\frac{\left(f / A^{*}\right) v_{2 s}{ }^{1 / 3}}{\alpha-\alpha^{-2}}
$$

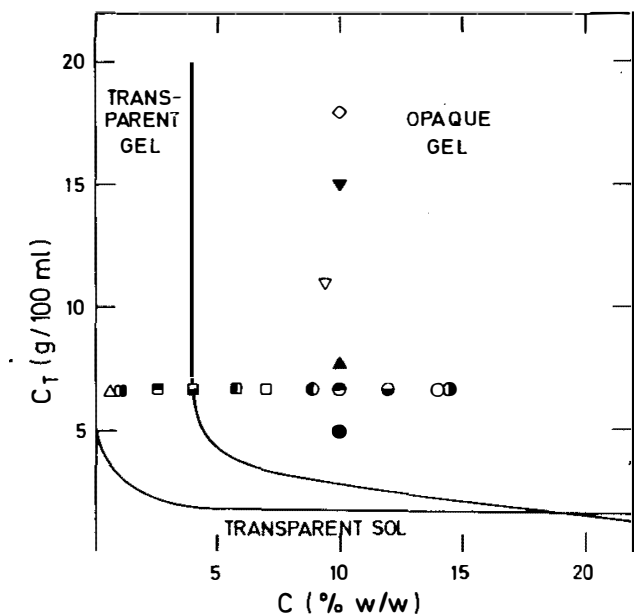

Figure 2. Phase diagram indicating the two sets of experiments at $C \simeq 10 \%$ and $C_{\mathrm{T}} \simeq 6.7 \mathrm{~g} / 100 \mathrm{~mL}$. Sample: $(\diamond) \mathrm{T}-1 ;(\nabla) \mathrm{T}-2$

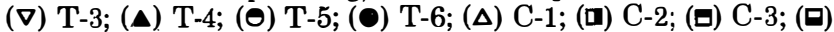
C-4; (घ) C-5; (ㅁ) C-6; (O) C-7; (○) C-9; (O) C-10; (o) C-11. For full details see Table I. The continuous lines are the boundaries between the different phases of PAA gels reported by Bansil and Gupta. ${ }^{10}$

where $f$ is the elastic force at equilibrium, $A^{*}$ is the cross-sectional area of the unstretched unswollen sample, and $v_{2 s}$ is the degree of swelling of the sample (gel volume fraction) at the given deformation $\alpha\left(\alpha=L / L_{0}, L\right.$ and $L_{0}$ being the lengths of the deformed and undeformed states, respectively). Since the measurements are carried out with the sample immersed in water, the degree of swelling changes with $\alpha$ ( $v_{23}$ decreases with elongation). Therefore it is necessary to correct $v_{2 s}$ at each measurement. As an approximation, the degree of swelling has been expressed by

$$
v_{2 \mathrm{~s}}=v_{2 \mathrm{~s}}{ }^{0} \alpha^{-3 / 5}
$$

This expression can be obtained easily from the usual equilibrium swelling formula, ${ }^{13}$ in the limit of high dilution. The error introduced by using this approximation instead 


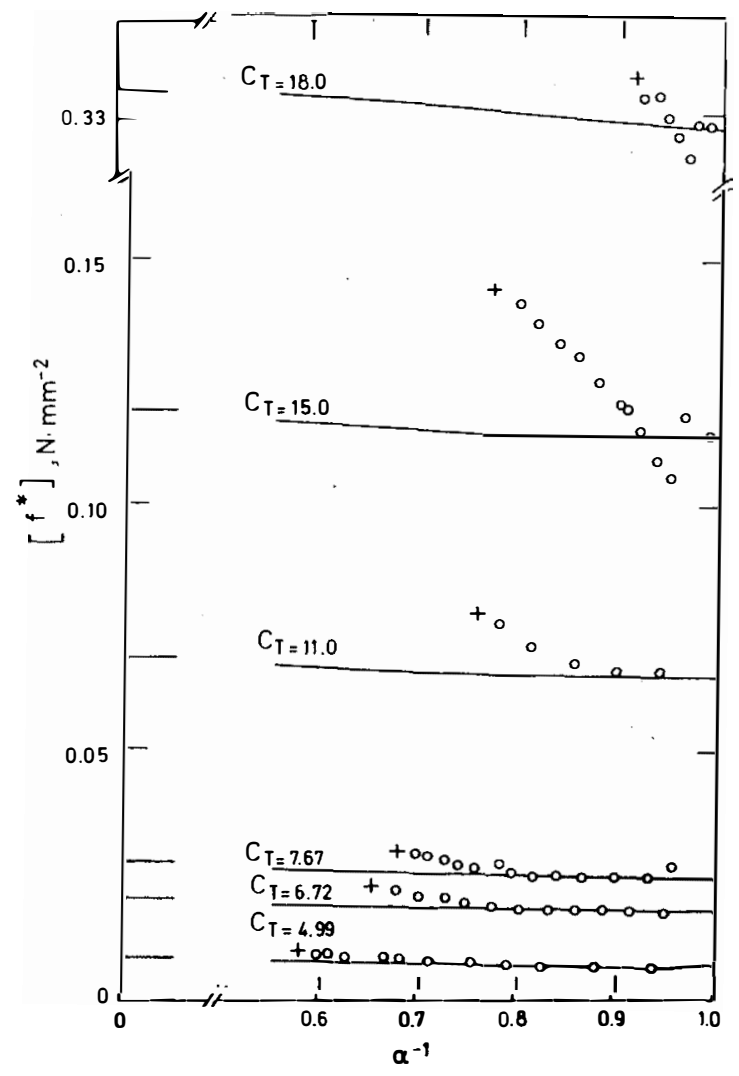

Figure 3. Reduced force versus $\alpha^{-1}$ for samples T-1 to T-6, at fixed cross-linker weight fraction $(C \simeq 10 \%)$. The crosses represent the rupture point for each experiment. The continuous lines are the Flory-Erman fitting curves, for which the limiting value at $\alpha^{-1} \rightarrow 0$ has been drawn representing the phantom modulus, $\left[f_{\mathrm{ph}}\right]$.

of the complete formula is less than $1 \%$ and it has no influence on the final results.

On the other hand, it is not possible to measure directly the cross-sectional area of the dried gel, so that it should be obtained from the one of the swollen sample $\left(A_{0}\right)$ assuming isotropic swelling, by means of the equation

$$
A^{*}=A_{0}\left(v_{28}{ }^{0}\right)^{2 / 3}
$$

As mentioned in the Experimental Section, two sets of gels were analyzed. Figure 3 shows the results obtained on the series of gels synthesized at constant cross-linker concentration $(C \simeq 10 \%)$. The data have been plotted as a function of $\alpha^{-1}$ in a Mooney-Rivlin form. As we can see the modulus increases as the total monomer concentration $\left(C_{\mathrm{T}}\right)$ increases. On the other hand, a deviation (upturn) from linearity takes place, this deviation being more pronounced at higher values of $C_{\mathrm{T}}$.

Due to this anomalous behavior of the data, the usual extrapolation procedures, such as the Mooney-Rivlin formulation, were not suitable for determination of the degree of cross-linking of the gels. Therefore we have employed the new Flory and Erman theory of rubber elasticity. ${ }^{14-16}$ In this approach the modulus is given by

$$
\left[f^{*}\right]=\left[f_{\mathrm{ph}}^{*}\right]\left[1+\left(f_{\mathrm{c}} / f_{\mathrm{ph}}\right)\right]
$$

where $\left[f^{*}{ }_{\mathrm{ph}}\right]$ is the modulus for the corresponding phantom network and $f_{\mathrm{c}}$ and $f_{\mathrm{ph}}$ are the contributions to the force from the constraints on fluctuations of junctions and from the equivalent phantom network, respectively. The ratio $f_{\mathrm{c}} / f_{\mathrm{ph}}$ is given by the theory as a function of two parameters, $k$, which is a measure of the severity of the constraints, and $\zeta$, which is related to the degree of nonaffineness in the relaxation of those constraints with strain. ${ }^{16}$
In order to interpret the results by the theory, the following extrapolation criterion has been used. The parameter $\kappa$ has been reported to be a function of the degree of crosslinking, ${ }^{17}{ }^{19}$ as expressed by the phantom modulus $\left[f^{*}{ }_{\mathrm{ph}}\right]$, according to the equation

$$
\kappa=I\left(\frac{N_{\mathrm{A}} d l^{2} C_{\infty}}{M_{0}}\right)^{3 / 2}\left(\frac{k T}{\left[f_{\mathrm{ph}}^{*}\right]}\right)^{1 / 2}\left(\frac{\phi-2}{\phi}\right)^{3 / 2}\left(\frac{2}{\phi-2}\right)
$$

where $I$ is a proportionality constant whose value has been taken to be $0.5,{ }^{17} N$ is Avogradro's number, $d$ is the density, $C_{\infty}$ is the characteristic ratio of the chains, $l$ and $M_{0}$ are their length and molecular weight per skeletal bond, $k$ is the Boltzmann constant, $\phi$ is the cross-linker functionality, and $T$ is the absolute temperature. For PAA chains the following parameters have been reported: $d=1.3004 \mathrm{~g} /$ $\mathrm{cm}^{3}{ }^{21} l=1.53 \AA, M_{0}=35.54 \mathrm{~g} / \mathrm{mol}$, and $C_{\infty}=8.5 .{ }^{20}$ From this set of parameters and the assumption $\phi=4$, the following relationship is found:

$$
\kappa=3.32\left[f^{*}{ }_{\mathrm{ph}}\right]^{-1 / 2}
$$

Therefore the data were fitted by adjusting simultaneously $\kappa$ and $\left[f^{*}{ }_{\mathrm{ph}}\right]$ along with the value of $\zeta$. This last parameter, though is not very important for unswollen networks and is usually set to zero, is really important for swollen gels. Setting this parameter to zero results in a poorer fit of the data since the theoretical lines have positive slopes at small deformations, whereas the experimental data indicate that the modulus increases with $\alpha$, giving negative slopes even for low values of $\alpha$.

In the isotherms of higher values of $C_{\mathrm{T}}$ it is not possible to fit properly the data even at small elongation ratios. These isotherms have been fitted with eq 6 and the value of $\zeta$ that gives the highest negative initial slope since this is the trend suggested by the data at lower values of $C_{\mathrm{T}}$.

As can be seen from Figure 3, the results can be fitted by the theory only at small elongations and lower values of $C_{\mathrm{T}}$. The pronounced upturns cannot be accounted for by the theory because they should be attributed to nonGaussian effects due to the increasing heterogeneities of the gels and these effects are not included in the theory. However, the theory is able to predict a small increase of the modulus with $\alpha$ in very highly diluted systems like the ones considered here. Other treatments like the Mooney-Rivlin formula would give horizontal lines at most, and the phantom modulus would have to be obtained at $\alpha \rightarrow 1$, assuming $2 C_{2}=0$ (very high dilution). The values are slightly lower than those calculated by Flory's theory but the final conclusions would be the same. In any case, the numerical values determined by us are in good agreement with previous data published in the literature obtained by different techniques. ${ }^{2,8}$ The results are given in columns 6 to 9 of Table I (gels T-1 to T-6). Values of the parameter $\kappa$ lie in the range expected, whereas $\zeta$ seems to decrease with decreasing total monomer concentration (decreasing modulus). The physical interpretation of this parameter has not been well established and it is usually related to the degree of imperfection of the network structure. In this regard the fact that $\zeta$ is higher for the gels of higher $C_{\mathrm{T}}$ suggests a higher heterogeneity.

Other important properties related to the gel structure are the ultimate properties, given in the last two columns of Table I; $\alpha_{\mathrm{r}}$ is the elongation ratio at the rupture point (denoted by a cross in Figure 3 ) and $\boldsymbol{E}_{\mathrm{r}}$ is the total energy needed for rupture of the sample, given by the total area under each curve of the force-deformation plot. $E_{\mathrm{r}}$ is therefore a measure of the "toughness" of the material. ${ }^{22,23}$ As expected $\alpha_{\mathrm{r}}$ decreases with increasing modulus due to 


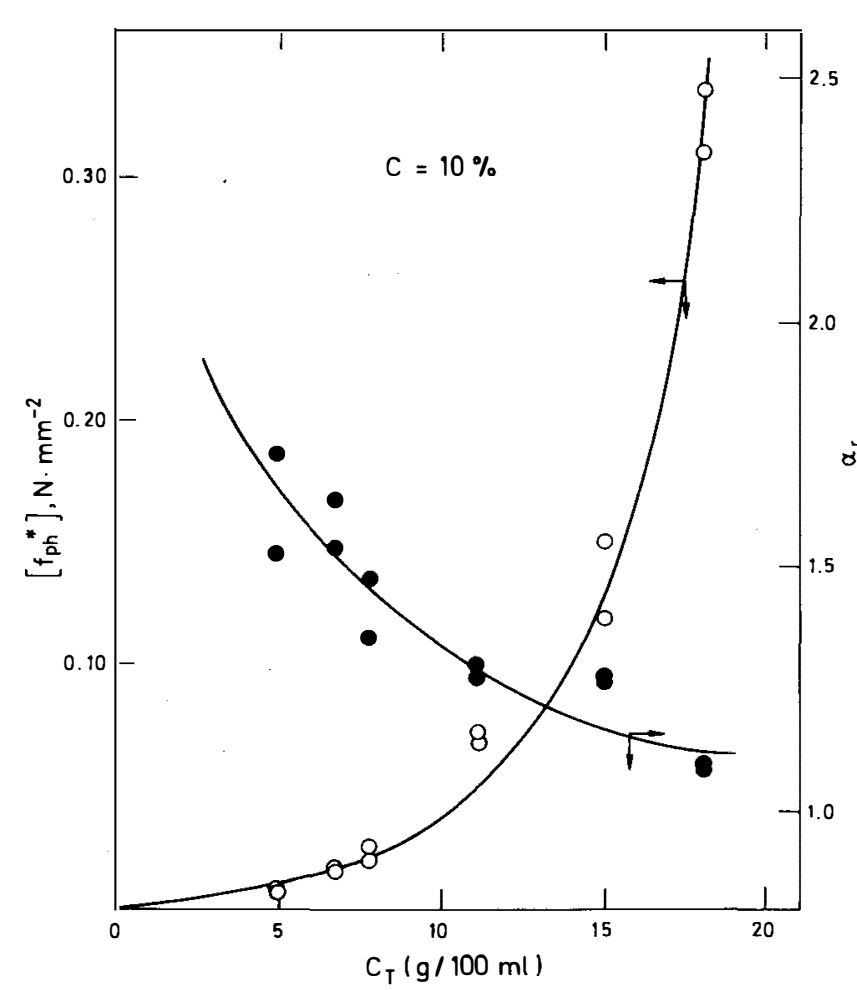

Figure 4. Phantom modulus, $\left[f^{*}\right](0)$, and rupture elongation, $\alpha_{\mathrm{r}}(\bullet)$, plotted as a function of the total monomer concentration $C_{\mathrm{T}}(\mathrm{g} / 100 \mathrm{~mL})$, for the samples T-1 to T-6 at fixed cross-linker weight fraction $(C \simeq 10 \%)$.

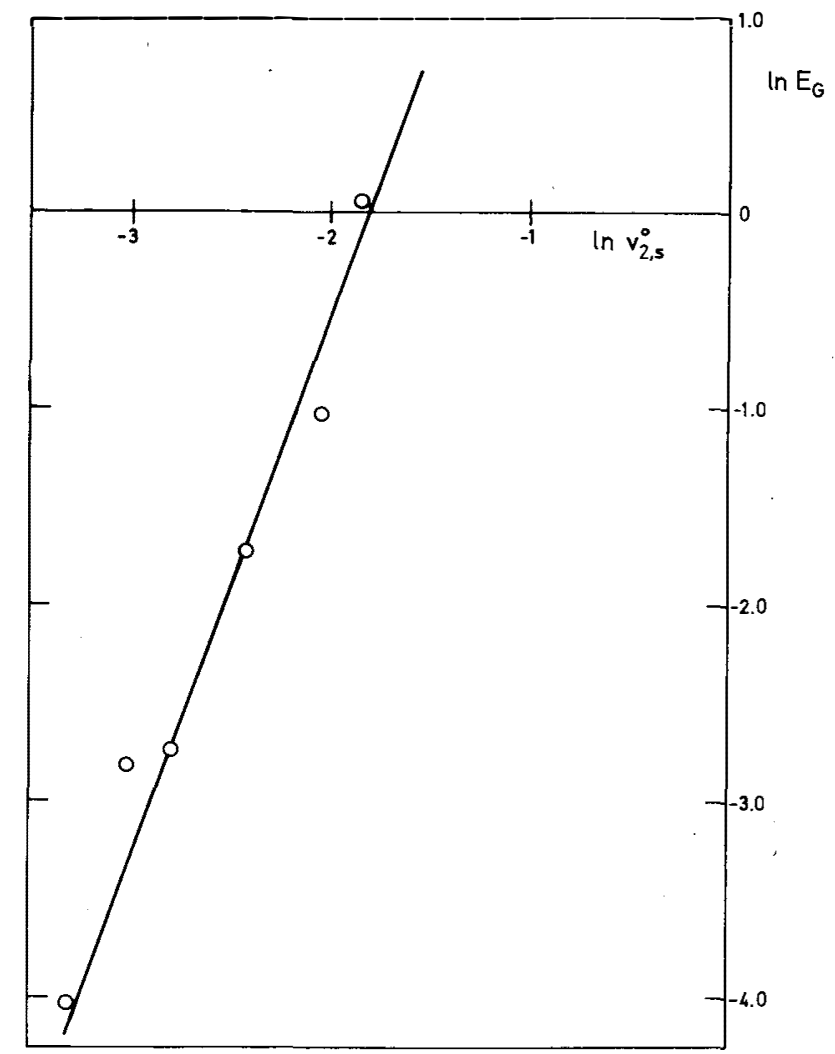

Figure 5. Shear modulus $\left(E_{\mathrm{G}}=1 /{ }_{3}\left[f_{\mathrm{ph}}^{*}\right]\right)$ of samples T- 1 to T-6 as a function of volume fraction, $v_{28}$, in the unstretched state.

the shortening of the network chains. Values of $E_{\mathrm{r}}$ are difficult to interpret due to the large errors involved. In general $E_{\mathrm{r}}$ seems to increase with total monomer concentration for values of $C_{\mathrm{T}}$ less than $15.0 \mathrm{~g} / 100 \mathrm{~mL}$.

In Figure 4, the phantom modulus and the elongation ratio at rupture for this set of gels are represented. As the

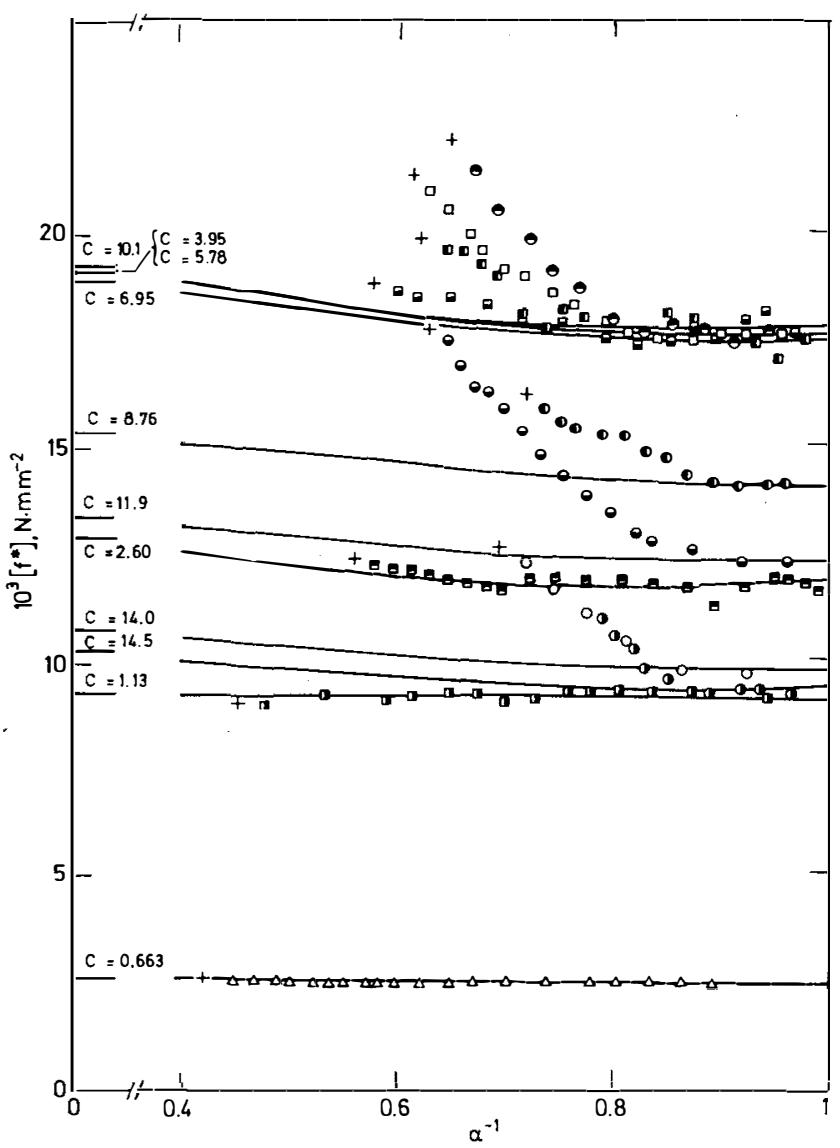

Figure 6. Reduced force versus $\alpha^{-1}$ for samples with constant $C_{\mathrm{T}} \simeq 6.7 \mathrm{~g} / 100 \mathrm{~mL}$ (samples C-1 to C-11 and T-5). Symbols for each sample correspond to the ones given in Figure 2. The crosses represent the rupture point for each experiment. For details about continuous lines see captions of Figure 3.

total monomer concentration increases $\alpha_{\mathrm{r}}$ decreases and the modulus grows exponentially. This variation of the modulus with polymer concentration agrees with predictions, based on scaling concepts, that give the following relationship for the shear modulus ${ }^{24,25}$

$$
E_{\mathrm{G}} \simeq\left(v_{2 s}{ }^{0}\right)^{3 \nu / 3 \nu-1}
$$

where $\nu$ is the excluded-volume exponent whose value should range between 0.588 (excluded-volume limit) and 0.5 ( $\theta$ conditions). ${ }^{26,27}$ Values of $E_{\mathrm{G}}$ obtained from our phantom modulus data $\left(E_{\mathrm{G}}=1 /{ }_{3}\left[f_{\mathrm{ph}}^{*}\right]\right)$ are plotted according to the logarithmic form of eq 7 in Figure 5 . As can be seen a good straight line is obtained with a slope of 2.55 which gives a value of $\nu=0.55$ for the excludedvolume exponent. This value lies in the range mentioned above and agrees with what should be expected for a good solvent.

The second series of results, obtained on gels synthesized by keeping the total monomer concentration constant is shown in Figures 6 and 7. As can be seen the behavior here is more complicated. Increasing BA monomer percentage first causes an increase in modulus but after $C=$ 5-6\% the modulus levels off, has a maximum, and then decreases (Figure 7). At the same time the upturn becomes more pronounced as $C$ increases (Figure 6 ), indicating a very high non-Gaussian effect on these gels. The numerical results are given also in Table I (gels C-1 to C-11). Values of the theoretical parameter $\zeta$ show no trend whatever and lie around 0.05. This is in contrast with the statement mentioned above that it should be higher for gels of higher heterogeneity. In any case, the fact that the results cannot be fitted properly by the theory, due to the strong non- 


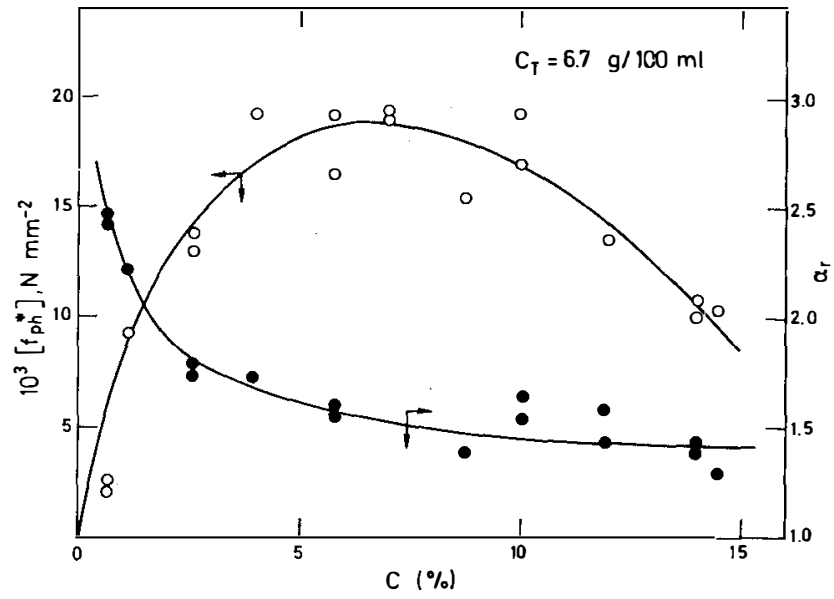

Figure 7. Phantom modulus, $\left[f^{*}{ }_{\mathrm{ph}}\right](0)$, and rupture elongation, $\alpha_{\mathrm{r}}(\bullet)$, plotted as a function of crosslinker weight fraction at fixed $C_{\mathrm{T}}\left(C_{\mathrm{T}} \simeq 6.7 \mathrm{~g} / 100 \mathrm{~mL}\right)$.

Gaussian effects found, keep open the physical interpretation of $\zeta$. The most important feature that can be drawn from Figure 7 is that there is a range of values of $C$ in which the mechanical properties are enhanced since the modulus has a maximum around $C=5-6 \%$ without loosing ultimate properties. Increasing further the percentage of cross-linking monomer does not improve the elastic properties of the gels.

Most of the previously reported studies on PAA gels have been carried out on gels corresponding to the "transparent" zone of the phase diagram (Figure 2, low values of $C$ ). With regard to the total monomer concentration, the modulus is reported to increase exponentially with $C_{\mathrm{T}}{ }^{5}$ as we have found here for gels of higher crosslinker monomer percentage (opaque gel zone). A more interesting feature is the variation with $C$. At low values of cross-linker monomer percentage, a linear relationship is always found. ${ }^{2,8}$ However, at high values of $C$ this linear proportionality breaks down $\mathrm{n}^{4,5}$ and the modulus has a maximum. This behavior is just the one we have found in our study. The region in which the modulus begins to level off coincides approximately with the transparent gel-opaque gel boundary.

All these features should be related to the molecular structure of the gels. It is well-known that PAA gels are very heterogeneous ${ }^{4,5,7,28-34}$ and a number of different models for their structure have been proposed..$^{5,7,28,29,32}$ All these models consider two different regions in the gel: a number of highly cross-linked aggregates or clusters linked together by a more lightly cross-linked gel, thus resulting in a two-phase structure. In previous studies on the kinetics of the gelation process, ${ }^{33,34}$ we have found that the structure of PAA gels is a consequence of the different reactivity of the comonomers, the cross-linker $\mathrm{BA}$ reactivity being higher than that of AA. The soluble polymer formed initially is very rich in BA; as the polymerization reaction proceeds, the new polymer which is being incorporated in the gel is progressively poorer in BA thus resulting in the heterogenous structure mentioned above that is responsible for the mechanical properties reported in this paper. If the amount of cross-linker monomer is high, a large number of microgels are formed, but if the dilution is high, there is not enough acrylamide monomer to link these particles by PAA chains. As a consequence, a low degree of cross-linking gel is obtained with a large number of structures which are not elastically effective. The formation of these inelastic structures, consisting preferentially on intramolecular cycles, has been already reported ${ }^{35}$ for other networks. The number of these cycles increases

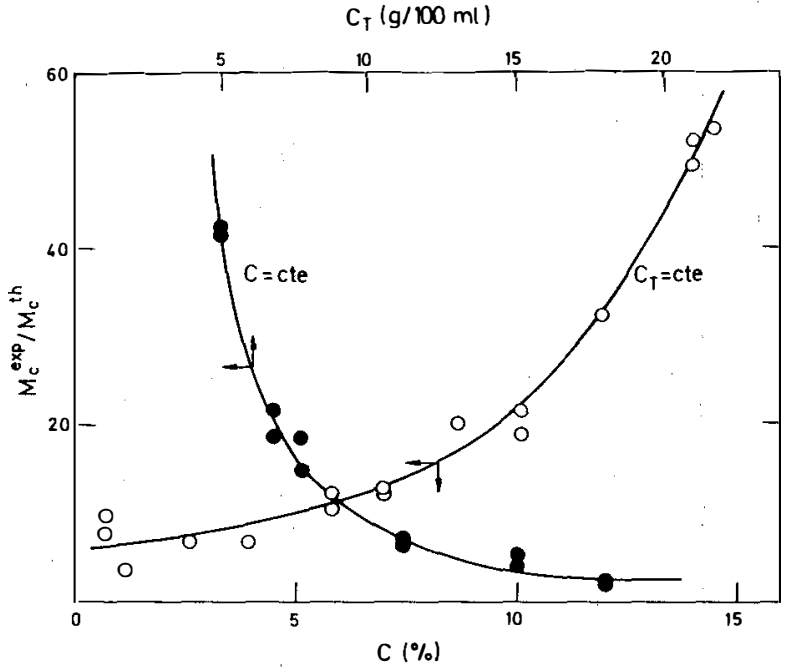

Figure 8. $M_{\mathrm{c}}{ }_{\mathrm{e}}^{\exp } / M_{\mathrm{c}}^{\text {th }}$ plotted as a function of $C_{\mathrm{T}}(\bullet)$ (samples T-1 to T-6) at constant $C(C \simeq 10 \%)$ and $C$ (O) (samples C-1 to C-11) at constant $C_{\mathrm{T}}\left(C_{\mathrm{T}} \simeq 6.7 \mathrm{~g} / 100 \mathrm{~mL}\right)$.

with dilution and with the proportion of cross-linking monomer.

The heterogeneous structure of these gels would also be responsible for the non-Gaussian effect found on their elastic properties. The presence of aggregates with a high degree of cross-linking would act as reinforcing particles (filler effect) thus preventing the premature rupture of the material. This same effect has been found on bimodal poly(dimethylsiloxane) networks ${ }^{22}$ which showed very good mechanical properties when a large concentration of short chains was present.

The existence of inelastic structures on PAA gels can be evaluated by comparison of the degree of cross-linking that can be calculated by the phantom moduli with the theoretical value obtained if the cross-linking process were ideal and the whole gel structure were elastic. From the phantom modulus, the "effective" molecular weight between cross-links, $M_{c}{ }^{\text {exp }}$, can be calculated by means of the equation ${ }^{36}$

$$
M_{\mathrm{c}}^{\exp }=(1-2 / \phi) \frac{d R T}{\left[f^{*}{ }_{\mathrm{ph}}\right]} \cdot\left(v_{2 \mathrm{c}}\right)^{2 / 3}
$$

The results are given in column 9 of Table I and they show the very large range of cross-linking densities covered by this study. On the other hand, the "theoretical" molecular weight, $M_{c}^{\text {th }}$, can be calculated by the equations

$$
\begin{gathered}
M_{\mathrm{c}}^{\text {th }}=\frac{[\mathrm{AA}] M_{\mathrm{AA}}}{2[\mathrm{BA}]} \\
{[\mathrm{AA}]=\frac{C_{\mathrm{T}}(100-C)}{10 M_{\mathrm{BA}}}} \\
{[\mathrm{BA}]=\frac{C_{\mathrm{T}} C}{10 M_{\mathrm{BA}}}}
\end{gathered}
$$

where $[\mathrm{AA}]$ is the concentration of $\mathrm{AA},[\mathrm{BA}]$ is the concentration of $\mathrm{BA}$, and $M_{\mathrm{AA}}$ and $M_{\mathrm{BA}}$ are their molecular weights, respectively.

Values of the ratio $M_{c}{ }_{c}^{\text {exp }} / M_{c}^{\text {th }}$ are given in column 10 of Table I. As can be seen these values may become very high indicating a high degree of inelastic structures for most of the samples. In Figure 8, this ratio is plotted for the two series of gels. The theoretical ideal value of 1 would only be obtained in the limit of very small dilution (high $C_{\mathrm{T}}$ ) or low percentage of cross-linker monomer (low C). 


\section{Concluding Remarks}

(i) Elastic modulus of PAA gels increases with total comonomer concentration, at a fixed cross-linker weight fraction, as expected from scaling theories; moreover, successive additions of cross-linker above 5\% do not improve the mechanical properties of the resulting gel; even more, they decrease, although this trend is not reflected on the ultimate properties (rupture elongation).

(ii) In the stress-strain isotherms, with either increasing $C_{\mathrm{T}}$ or $C$ the upturn on the modulus becomes more pronounced, indicating a bimodal structure of the PAA gels. This non-Gaussian effect can be related to the previously reported heterogeneous structure of PAA gels.

(iii) The high values of the ratio $M_{c}^{\exp } / M_{c}^{\text {th }}$, which is a measure of the cross-linking effectiveness and, therefore, of the network perfection, indicate a great degree of topological imperfections and inelastic structures. "Ideal" networks would be obtained only in the limits of high concentration and very low cross-linker percentage.

Acknowledgment. We thank Dr. P. Deporcellinis for his kind cooperation. This work was supported in part by Grant 0528/84 from the Comision Asesora de Investigacion Cientifica y Técnica.

Registry No. (Acrylamide)( $N, N^{\prime}$-methylenebisacrylamide) (copolymer), 25034-58-6.

\section{References and Notes}

(1) (a) Helbling, G. Eur. Pat. Appl. 37138 . (b) Raymond, S.; Weintraub, L. Science (Washington, D.C.) 1959, 130, 711. (c) Kuleshov, I. V.; Ksenofontov, B. S.; Shkop, Y. Modif. Polim. Mater. 1980, No. 9, 148.

(2) Nossal, R. Macromolecules 1985, 18, 49.

(3) Janas, V. F.; Rodriguez, F.; Cohen, C. Macromolecules 1980, $13,977$.

(4) Hsu, T. P.; Ma, D. S.; Cohen, C. Polymer 1983, 24, 1273.

(5) Weiss, N.; Silberberg, A. Br. Polym. J. 1977, 9, 144.

(6) Ulbrick, V. F.; Ilavsky, M.; Dusek, K.; Kopecek, J. Eur. Polym. J. 1977, 13, 579 .

(7) Richards, E. G.; Temple, C. J. Nature (London) Phys. Sci. $1977,22,92$
(8) Opperman, W.: Rose, S.; Rehage, G. Br. Polym. J. 1985, 17, 175.

(9) Huglin, M. B.; Rehab, M. M. A. M.; Zakaria, M. B. Macromolecules 1986, 19, 2986.

(10) Bansil, R.; Gupta, M. K. Ferroelectrics 1980, 30, 63.

(11) Mark, J. E. In Elastomers and Rubber Elasticity; Mark, J. E. Lal, J., Eds.; American Chemical Society: Washington, DC, 1982.

(12) Mark, J. E.; Sullivan, J. L. J. Chem. Phys. 1977, 66, 1006.

(13) Flory, P. J. Principles of Polymer Chemistry; Cornell University: Ithaca, NY, 1953; Chapter 13.

(14) Flory, P. J. Br. Polym. J. 1985, 17, 96.

(15) Flory, P. J. J. Chem. Phys. 1977, 66, 5720.

(16) Flory, P. J.; Erman, B. Macromolecules 1982, 15, 800.

(17) Erman, B.; Flory, P. J. Macromolecules 1982, 15, 808.

(18) Brotzman, R. N.; Mark, J. E. Macromolecules 1986, 19, 667.

(19) Sanjuan, J.; Llorente, M. A. J. Polym. Sci., Polym. Phys. Ed., in press.

(20) Bohdanecky, M.; Petrus, V.; Sedlácek, B. Makromol. Chem. 1983, 184, 2061.

(21) Scholtan, W. Makromol. Chem. 1954, 14, 169

(22) Llorente, M. A.; Andrady, A. L.; Mark, J. E. J. Polym. Sci., Polym. Phys. Ed. 1981, 19, 621.

(23) Mark, J. E.; Tang, M.-Y. J. Polym. Sci., Polym. Phys. Ed. $1984,22,1849$.

(24) De Gennes, P.-G. Scaling Concepts in Polymer Physics; Cornell Univesity Press: Ithaca, NY, 1979.

(25) Horkay, F.; Zrinyi, M. Macromolecules 1982, 15, 1306.

(26) Le Guillon, J. C.; Zinn-Justin, J. J. Phys. Rev. Lett. 1977, 39 , 95.

(27) Horkay, F.; Zrinyi, M. Acta Chim. Hung. 1983, 114, 26.

(28) Hsu, T. P.; Cohen, C. Polymer 1984, 25, 1419.

(29) Gupta, M. K.; Bansil, R. J. Polym. Sci., Polym. Lett. Ed. 1983, 21,969 .

(30) Geissler, E.; Hecht, A. M.; Duplessix, R. J. Polym. Sci., Polym. Phys. Ed. 1982, 20, 225

(31) Weiss, N. Polymer Science and Technology; 16, Cooper, A. R., Ed.; Plenum Press: New York, 1981; Vol. 16, p 247.

(32) (a) Weiss, N.; Van Vliet, T. T.; Silberberg, A. J. Polym. Sci., Polym. Phys. Ed. 1981, 19, 1505. (b) Ibid. 1979, 17, 2229.

(33) Nieto, J. L.; Baselga, J.; Hernändez-fuentes, I.; Llorente, M. A.; Piêrola, I. F. Eur. Polym. J., in press.

(34) Baselga, J.; Hernändez-Fuentes, I.; Piêrola, I. F.; Llorente, M. A. Eur. Polym. J., in press.

(35) Dusek, K.; Galina, H.; Mikes J. Polym. Bull. 1980, 3, 19.

(36) Andrady, A. L.; Llorente, M. A. J. Polym. Sci., Polym. Phys. Ed. 1987, 25, 195. 